\section{Perfil de exposição ao chumbo em crianças escolares da rede municipal da cidade do Rio de Janeiro, Brasil}

\section{The lead exposure profile of municipal public schoolchildren in the city of Rio de Janeiro, Brazil}

Maíra Lopes Mazoto 1

Raphael Mendonça Guimarães 2

Volney Magalhães Câmara 3

Carmen Ildes Rodrigues Fróes Asmus 4

1-4 Instituto de Estudos em Saúde Coletiva. Universidade Federal do Rio de Janeiro. Praça Jorge Machado Moreira, s.n. Cidade Universitária. Ilha do Fundão. Rio de Janeiro, RJ, Brasil. CEP: 21.944970. E-mail: mairamazoto@iesc.ufrj.br

\begin{abstract}
Objectives: to describe the levels of environmental exposure to lead among children from two schools in regions with distinct social and environmental features in the municipality of Rio de Janeiro.

Methods: a pilot study was carried out in a convenience sample of 270 students. Analysis of capillary blood samples was conducted using the Inductively Coupled Plasma Mass Spectrometry (ICP-MS). Lead exposure among the children was estimated on the basis of the their daily habits and behavior patterns and the presence of sources of lead in the household or surrounding area.

Results: the mean concentration of lead in capillary blood was $3.59 \mu \mathrm{g} / \mathrm{dL} \pm 2.25$. Five children (1.85\%) had concentrations higher than $10 \mu \mathrm{g} / \mathrm{dL}$. A statistically significant difference was found in the mean concentration of lead in capillary blood for the variables age $(p=0.034)$, per capita income $(p=0.005)$ and exposure to lead $(p=0.044)$. The variables mean household per capita income and age were inversely proportional to the concentration of lead and exposure status directly proportional. There was a statistically significant difference between the schools ( $p=0.004)$ in terms of the prevalence of children exposed to lead.

Conclusions: although the school variable was not a good predictor of capillary concentrations of lead, the higher prevalence of children exposed to lead was found in the school situated in the most polluted area.
\end{abstract}

Key words Epidemiology, Environmental health, Environmental exposure, Lead, Child

\section{Resumo}

Objetivos: descrever os niveis de exposição ambiental ao chumbo entre criancas de duas escolas localizadas em regiões com características socioambientais distintas no município do Rio de Janeiro.

Métodos: estudo piloto em amostra de conveniência com 270 estudantes. A análise das amostras de sangue capilar foi realizada usando o método de Espectrometria de Massa Acoplado a Plasma Indutivo (ICP-MS). A exposição ao chumbo entre as crianças foi estimada através de comportamentos e habitos diarios e presença de fontes de chumbo no domicilio ou entorno.

Resultados: a concentração média do chumbo no sangue capilar foi 3,59 $\mu \mathrm{g} / \mathrm{dL} \pm 2,25$. Cinco crianças (1,85\%) apresentaram concentrações acima de 10 $\mu \mathrm{g} / \mathrm{dL}$. Observou-se uma diferença estatisticamente significativa na concentração média de chumbo em sangue capilar nas variáveis idade $(p=0,034)$, renda per capita $(p=0,005)$ e exposição a chumbo $(p=0,044)$. As variáveis renda média familiar per capita e idade mostraram-se inversamente proporcionais à concentração de chumbo e o status de exposição diretamente proporcional. As escolas apresentaram diferença estatisticamente significativa $(p=0,004)$ quanto à prevalência de crianças expostas ao chumbo.

Conclusões: embora a variável escola não tenha sido boa preditora da concentração capilar de chumbo, a maior prevalência de crianças expostas a chumbo se encontra na escola localizada na área mais poluida.

Palavras-chave Epidemiologia, Saúde ambiental, Exposição ambiental, Chumbo, Criança 


\section{Introdução}

O chumbo é um metal tóxico, não-essencial, amplamente distribuído no ambiente e absorvido, principalmente, pelas vias respiratória e gastrintestinal. Pela sua ocorrência natural no ambiente e sua ampla utilização na indústria, é considerado um metal onipresente na natureza, ao qual os seres humanos em geral encontram-se expostos de forma ocupacional e não-ocupacional. 1

Embora as intoxicações provocadas pelo chumbo na população humana sejam causadas, principalmente por exposição ocupacional, o metal é particularmente tóxico para as crianças que apresentam particular suscetibilidade aos seus efeitos adversos devido as suas características fisiológicas e hábitos.2,3 Quando expostas, as crianças absorvem maior quantidade de chumbo quando comparadas aos adultos 4 e como a barreira hematoencefálica e o sistema imunológico ainda estão em desenvolvimento, tornando-as mais vulneráveis à exposição ao chumbo. Além disso, as crianças possuem hábitos, como brincar com terra e levar a mão na boca, que as tornam mais vulneráveis à exposição a este metal quando comparadas aos adultos. $2-5$

A exposição ambiental ao chumbo, mesmo em baixas concentrações, exerce efeitos negativos no organismo infantil. 4 Estudos mostram que o chumbo pode ocasionar danos ao sistema cardiovascular, causando aumento da pressão arterial (concentrações de chumbo sanguíneo $>40 \mu \mathrm{g} / \mathrm{dL}$ ), ${ }^{6}$ redução da velocidade de crescimento (concentrações de chumbo sanguíneo superiores a $20 \mu \mathrm{g} / \mathrm{dL}) ; 7$ anemia (níveis de chumbo em sangue iguais a $25 \mu \mathrm{g} / \mathrm{dL}$ ); 8 retardo do desenvolvimento neuropsicológico (entre 10 e $5 \mu \mathrm{g} / \mathrm{dL}$ ), cognitivos e de memória, além de interferir negativamente no desenvolvimento emocional e comportamental e no quociente de inteligência (QI) (níveis inferiores a $5 \mu \mathrm{g} / \mathrm{dL}$ ) das crianças. 5

Este estudo teve por objetivo descrever os níveis de exposição ambiental ao chumbo de estudantes de duas escolas localizadas em regiões com características socioambientais distintas no município do Rio de Janeiro, no período de 2008 a 2011.

\section{Métodos}

Foi realizado um estudo piloto observacional, de corte seccional, em crianças matriculadas em duas escolas da rede municipal de ensino da cidade do Rio de Janeiro localizadas em regiões com características socioambientais distintas no período de 2008 a 2011. As regiões se distinguem por características socioambientais. Observa-se, por exemplo, que Bonsucesso possui uma maior densidade demográfica, uma menor concentração de área verde preservada, e uma qualidade do ar considerada inadequada, graças à intensa atividade industrial na área. Estas características possuem relevância para compreender a dispersão do chumbo no ambiente e suas prováveis rotas de contaminação. 1

As unidades escolares do município do Rio de Janeiro são divididas em Coordenadorias Regionais de Educação (CRE). Cada CRE é composta por 3035 bairros e, consequentemente, entre 140-180 unidades escolares.

Para a seleção do local do estudo, os bairros foram divididos em função de suas condições socioambientais. Além disso, as escolas escolhidas deveriam, obrigatoriamente, obedecer os seguintes critérios pré-estabelecidos: possuírem 300 ou mais crianças com idades entre oito e dez anos, regularmente matriculadas no ano de 2007; segurança para o acesso dos pesquisadores; aquiescência da direção e professores, existência de um Programa de Saúde Escolar.

A seleção do local de estudo foi realizada por conveniência, tendo sido definida pela Secretaria Municipal de Educação do Rio de Janeiro, a qual indicou as $4^{\mathrm{a}}$ e a $7^{\mathrm{a}}$ Coordenadorias Regionais de Educação (CRE). Assim, as escolas municipais selecionadas foram o CIEP Yuri Gagarin, localizado no bairro de Bonsucesso, e a Escola Desembargador Ney Palmeiro, localizada no bairro de Jacarepaguá, regiões com situações socioambientais distintas (Tabela 1).

Duzentos e setenta escolares com idade entre oito e dez anos participaram do estudo. O convite para a participação foi realizado em uma reunião com os pais e responsáveis legais pelas crianças, além de professores e dos próprios alunos, na qual foram apresentados o desenho e os objetivos da pesquisa.

Após leitura e assinatura dos Termos de Consentimento Livre e Esclarecido (TCLE) pelos pais ou responsáveis, realizou-se a coleta dos dados, que envolveu a realização de entrevistas com as crianças e familiares utilizando questionários padronizados e testados, para obtenção de dados individuais da criança (dados pessoais, hábitos alimentares, tabagismo, ocupação dos moradores da residência, moradia e ocorrência de menarca) e da família (informações sobre os pais ou responsáveis: dados pessoais, hábitos alimentares, ocupação; informações sobre a família: endereço, telefone, número de pessoas nas casas; informações sobre a criança: quanto tempo mora no local, se já morou em outro 
Caracterização das áreas estudadas.

\begin{tabular}{|c|c|c|}
\hline \multirow[t]{2}{*}{ Características } & \multicolumn{2}{|c|}{ Município } \\
\hline & Bonsucesso & Jacarepaguá \\
\hline Área (hectares) & 3712 & 12.771 \\
\hline População residente (habitantes) & 475.738 & 507.698 \\
\hline Áreas naturais (\%) & 1.55 & 61.06 \\
\hline Áreas urbanizadas e/ou alteradas (\%) & 98.45 & 38.94 \\
\hline $\begin{array}{l}\text { Proporção do segmento de comércio } \\
\text { e serviços }(\%)\end{array}$ & 78,7 & 88,4 \\
\hline Volume de negócios & $\begin{array}{c}\text { Gera } \mathrm{R} \$ 335,9 \text { milhões de ICMS } \\
\text { (US\$289,4 milhões) }\end{array}$ & $\begin{array}{c}\text { Gera R\$ } 439 \text { milhões de ICMS } \\
\text { (US\$378,3 milhões) }\end{array}$ \\
\hline $\begin{array}{l}\text { Ranking de arrecadação entre as } 12 \\
\text { regiões }\end{array}$ & $4^{\mathrm{a}}$ & $2^{\mathrm{a}}$ \\
\hline Desenvolvimento humano (IDH) & Médio-alto (IDH=0,777) & Médio-alto $(\mathrm{IDH}=0,800)$ \\
\hline Renda média (salários mínimos) & 3 & 5 \\
\hline Taxa de alfabetização (\%) & 91 & 93 \\
\hline População com escolaridade superior & $10,6 \%$ da população residente & Cerca de $16 \%$ da população residente \\
\hline Qualidade do ar & Inadequada & Boa \\
\hline
\end{tabular}

Fontes: Prefeitura da Cidade do Rio de Janeiro, 2003; INEA, 2009.

local, se já morou próximo a lixão, se possui alguma doença, se toma algum medicamento, hábitos alimentares: se come peixe, vísceras/miúdos, frutosdo-mar; informações sobre a residência da criança: se a casa foi reformada/pintada recentemente, tipo de asfaltamento da rua, tipo de água para consumo humano: galão, poço ou nascente, se possui criação de animais em casa, atividades perto da residência).

A coleta de sangue foi realizada através de punção digital com retirada de 5-10 $\mathrm{mL}$ de sangue capilar.

As amostras coletadas foram acondicionadas em caixas térmicas para transporte do local da coleta (Escola) armazenadas em laboratório, em freezer à temperatura de $-20^{\circ} \mathrm{C}$ até serem enviadas para a análise.

A análise das amostras foi realizada no Laboratório de Toxicologia de Metais da Universidade de São Paulo (USP), na cidade de Ribeirão Preto utilizando o método de Espectrometria de Massa Acoplado a Plasma Indutivo (ICP-MS). Após o descongelamento, as amostras de sangue foram diluídas na proporção de
1:50, em tubo de polipropileno, com uma solução $0,5 \%(\mathrm{v} / \mathrm{v})$ de ácido nítrico contendo $0,01 \%(\mathrm{v} / \mathrm{v}) \mathrm{de}$ Triton X-100. O amoníaco foi usado como gás de reação. O limite de detecção do método para o chumbo é de $82 \mathrm{ng} / \mathrm{L}$. A validação do método foi realizada através da análise de referência para sangue produzidas pelo L'Institut National de Santé Publique du Quebec, Canadá. ${ }^{9}$ Os resultados obtidos foram dados em ng/L.

$\mathrm{Na}$ análise as variáveis de exposição consideradas no estudo: atividade da criança (artesanato, cerâmica, pintura, cerol); brinca/come terra, comeu vísceras, carne, frango e frutos-do-mar; moradia (se na casa tem posto de gasolina, fabrica de moveis, fabrica de fogos de artifício e munição, oficina de conserto de carros, e outros); perto da casa tem (se perto da casa tem: tem posto de gasolina, fabrica de moveis, fabrica de fogos de artifício e munição, oficina de conserto de carros e outros); trabalho (ocupação dos moradores da casa); trabalho do pai; trabalho da mãe; tratamento de cabelo; morou próximo a lixão. Como perguntas consideradas chave para o estudo apareciam em ambos os ques- 
tionários, as variáveis moradia, perto de casa tem e trabalho, foram subdivididas em moradia e moradia família, perto de casa tem e perto de casa tem família e trabalho e trabalho família, uma referindo-se ao questionário criança e a outra refere-se ao questionário família, respectivamente. Assim, chegou-se a um total de 12 (doze) variáveis de exposição no estudo.

Todas as variáveis de exposição foram variáveis categóricas dicotômicas, respondendo às opções sim e não. Algumas das variáveis foram agregadas, de forma a descrever o padrão de exposição de acordo com a potencial via de absorção do chumbo. A variável atividade criança foi o resultado da presença positiva de alguma das características atividade pintura + atividade cerâmica + atividade artesanato + cerol (portanto, com uma provável via de absorção dérmica). Criou-se a variável brincacomecomeu, caso tenha positivado alguma das seguintes questões: brinca com terra + come terra/lápis + comeu vísceras, frango, carne, frutos-do-mar (portanto, com via de absorção oral). As variáveis relativas à ocupação dos moradores da casa (fabrica/conserto de baterias de carro + fabrica de tintas + oficina de conserto de carros + fabricação de potes de barro + fabrica de munição para armas de fogo + fabricação de moveis + fundição/metalurgia/ ferro velho + fazer chumbada em casa para pescaria) foram agregadas e renomeadas para trabalho, para descrever uma provável absorção via inalatória. Ainda, a variável características da moradia (na sua casa tem) deu origem a moradia (estamparia de camisetas + produção de detergentes + cabeleireiro + oficina de conserto de carros + fabricação de potes de barro (cerâmica) + artesanato + fabricação de moveis + fundição/metalurgia/ferro velho), para uma via de exposição inespecífica.

Desta forma, o escore de exposição das crianças foi obtido através do somatório simples das médias das variáveis recodificadas descritas acima. Finalmente, para classificar as populações, o escore de exposição foi categorizado de acordo com a mediana.

A variável idade foi tratada como variável categórica, uma vez que havia crianças apenas com três opções de idade, devido ao público alvo: 8,9 e 10 anos.

A variável renda foi tratada considerando a renda per capita, obtida através da divisão entre a renda total e o número de pessoas que dependiam daquela renda e, em seguida, a população foi classificada de acordo com a mediana da renda per capita em baixa renda per capita e alta renda per capita.

Foram estimadas as médias da concentação de chumbo capilar para cada estrato das variáveis selecionadas como preditoras: idade, sexo, escola, renda média per capita e status de exposição. Em seguida, para as variáveis com diferença de médias estatisticamente significativas, foi gerado um modelo de regressão linear mútipla, tendo como variável desfecho a concentração capilar de chumbo, padronizada de acordo com a seguinte fórmula:

$$
\frac{\mathrm{z}=(\mathrm{X}-\mu)}{\sigma}
$$

Sendo:

$$
\begin{aligned}
& X=\text { concentração capilar de chumbo } \\
& \mu=\text { média populacional } \\
& \sigma=\text { desvio padrão populacional }
\end{aligned}
$$

A análise dos dados foi feita utilizando programa SPSS, versão 21.0. Foram realizadas análises descritivas das médias e percentagens das concentrações de chumbo na população de estudo.

O teste não paramétrico de Kruskal-Wallis foi utilizado para determinar a relação entre as concentrações de chumbo em sangue capilar e as variáveis descritivas idade e renda total da família. A população de estudo foi classificada segundo quartis de distribuição dos níveis de concentração de chumbo em sangue capilar e foi realizada uma análise bivariada com as variáveis de exposição com seus respectivos escores. Foi aplicado o teste quiquadrado de Pearson para determinar as diferenças entre as variáveis de exposição com seus respectivos escores e as concentrações de chumbo em sangue capilar na população estudada.

O presente estudo respeitou os procedimentos éticos da Declaração de Helsinque e da Resolução $n^{\circ}$ 196/96 do Conselho Nacional de Saúde sobre pesquisa envolvendo seres humanos e foi submetido e aprovado pelo Comitê de Ética do Instituto de Estudos em Saúde Coletiva (IESC) (parecer $\mathrm{n}^{\circ}$ 45/2008).

\section{Resultados}

Quinhentas e vinte e duas crianças foram consideradas elegíveis para o estudo nas duas escolas selecionadas. Trezentos e trinta e oito Termos de Consentimento foram assinados e 314 amostras foram coletadas. Uma perda total de $14 \%$ nos processos de coleta, armazenamento e análise.

A população de estudo foi composta por 270 crianças, oriundas de duas escolas públicas do município do Rio de Janeiro, com média de idade foi $9,2 \pm 0,8$ anos. A maioria era do sexo feminino $(56,7 \%)$ de cor não branca $(63,3 \%)$ (Tabela 2). Observou-se uma diferença estatisticamente signi- 
ficativa entre as escolas estudadas apenas em relação a distribuição por sexo $(p=0,039)$ e estado de nascimento ( $p=0,032)$, havendo um maior número percentual de crianças do sexo masculino na Escola 1 do que na Escola 2. Considerando que estas duas variáveis não interferem no padrão de exposição para crianças, as populações das duas escolas são comparáveis.

A concentração média de chumbo encontrada no presente estudo foi de 3,59 $\mu \mathrm{g} / \mathrm{dL}(\mathrm{DP} \pm 2,25)$.

As concentrações médias de chumbo no sangue capilar encontradas nas escolas estudadas foram de $3,57 \mu \mathrm{g} / \mathrm{dL} \pm 2,74$ na Escola 1 (Bonsucesso) e de $3,59 \mu \mathrm{g} / \mathrm{dL} \pm 1,92$ na escola 2 (Jacarepaguá), não havendo diferença estatisticamente significativa $(p=0,496)$ entre as escolas. Observou-se uma diferença estatisticamente significativa na concentração média de chumbo em sangue capilar em relação às variáveis idade $(p=0,034)$, renda per capita $(p=0,005)$ e exposição a chumbo $(p=0,044)$. As crianças com menor renda per capita familiar apresentaram concentrações de chumbo em sangue capilar superiores às encontradas nas crianças com renda familiar per capita superior. Além disso, crianças mais jovens, com 8 anos, possuem uma média de concentração de chumbo notadamente maior que as crianças de 9 e 10 anos. Por fim, as crianças classificadas como sendo expostas, de acordo com o escore obtido através de hábitos de ingesta e contato com potenciais rotas de exposição apresentam concentração média maior do que as crianças classificadas como não expostas. (Tabela 3).

Tomando as variáveis significativas na Tabela 3 (idade, renda média familiar per capita e status de exposição), procedeu-se à modelagem de regressão linear múltipla. Obteve-se um modelo significativo $(p=0,01)$, com alto coeficiente de determinação $\left(\mathrm{R}^{2}=0,61\right)$, e parâmetros apresentados na Tabela 4 . As variáveis renda média familiar per capita e idade apresentaram-se inversamente proporcionais à concentração de chumbo, e a variável status de exposição apresentou-se diretamente proporcional à variável desfecho. Cabe ressaltar que, realizada a análise de resíduos, todos os pressupostos (aleatoriedade, independência, heterocedasticidade e normalidade) foram atendidos, de forma que o

Tabela 2

Análise descritiva da população de estudo. Rio de Janeiro, $2011(\mathrm{~N}=270)$.

\begin{tabular}{|c|c|c|c|c|c|c|c|}
\hline \multirow[t]{2}{*}{ Variáveis } & \multirow[t]{2}{*}{$\mathbf{N}$} & \multirow[t]{2}{*}{$\%$} & \multicolumn{2}{|c|}{ Escola 1} & \multicolumn{2}{|c|}{ Escola 2} & \multirow[t]{2}{*}{$p$} \\
\hline & & & $\mathrm{n}$ & $\%$ & $\mathrm{n}$ & $\%$ & \\
\hline Idade (anos) & 270 & & 99 & 36,6 & 171 & 63,4 & 0,118 \\
\hline 8 & 65 & 24,1 & 24 & 24,2 & 41 & 24,0 & \\
\hline 9 & 90 & 33,3 & 40 & 40,4 & 50 & 29,3 & \\
\hline 10 & 115 & 42,6 & 35 & 35,4 & 80 & 46,7 & \\
\hline Sexo & & & & & & & 0,039 \\
\hline Feminino & 153 & 56,7 & 48 & 48,5 & 105 & 61,4 & \\
\hline Masculino & 117 & 43,3 & 51 & 51,5 & 66 & 38,6 & \\
\hline Tempo de residência (meses) & & & & & & & 0,014 \\
\hline $0-12$ & 40 & 14,8 & 15 & 15,2 & 25 & 14,6 & \\
\hline $13-60$ & 65 & 24,1 & 28 & 28,3 & 37 & 21,6 & \\
\hline Mais 60 & 131 & 48,5 & 37 & 37,4 & 94 & 55,0 & \\
\hline Estado de nascimento & & & & & & & 0,032 \\
\hline Rio de Janeiro & 233 & 86,3 & 79 & 79,8 & 154 & 90,0 & \\
\hline Outros & 36 & 13,3 & 20 & 20,2 & 16 & 9,3 & \\
\hline Moradores por domicílio & & & & & & & 0,140 \\
\hline $0-5$ & 142 & 52,6 & 43 & 43,3 & 99 & 58,0 & \\
\hline $6-10$ & 125 & 46,3 & 53 & 53,5 & 72 & 42,0 & \\
\hline$>10$ & 3 & 1,1 & 3 & 3,0 & 0 & - & \\
\hline Renda per capita (salários mínimos) & & & & & & & 0,269 \\
\hline Até 1,3 SM (baixa renda) & 175 & 64,8 & 67 & 67,7 & 108 & 63,2 & \\
\hline Acima de 1,3 SM (alta renda) & 95 & 35,2 & 32 & 32,3 & 63 & 36,8 & \\
\hline
\end{tabular}

Escola 1= CIEP Yuri Gagarin (Bonsucesso); Escola 2= Escola Desembargador Ney Palmeiro (Jacarepaguá). 
modelo mostrou-se adequado.

Embora a variável escola não tenha sido considerada preditora da concentração capilar de chumbo, observou-se a prevalência de pessoas classificadas como expostas em cada uma delas, e obteve-se que a escola localizada em Bonsucesso (CIEP Yuri
Gagarin), a região com maior poluição ambiental, concentrava mais crianças classificadas como expostas. A diferença na prevalência foi estatisticamente significativa $(p=0,004)$, conforme apresentado na Tabela 5.

Tabela 3

Concentrações médias de chumbo em sangue capilar na população distribuídas pelas variáveis descritivas do estudo. Rio de Janeiro, 2011.

\begin{tabular}{lllll}
\hline Variáveis descritivas & $N$ & $\begin{array}{l}\text { Concentração } \\
\text { média de } \mathrm{Pb}-\mathrm{S} \\
\text { capilar }(\mu \mathrm{g} / \mathrm{dL})\end{array}$ & DP & Mediana \\
& & \\
\hline
\end{tabular}

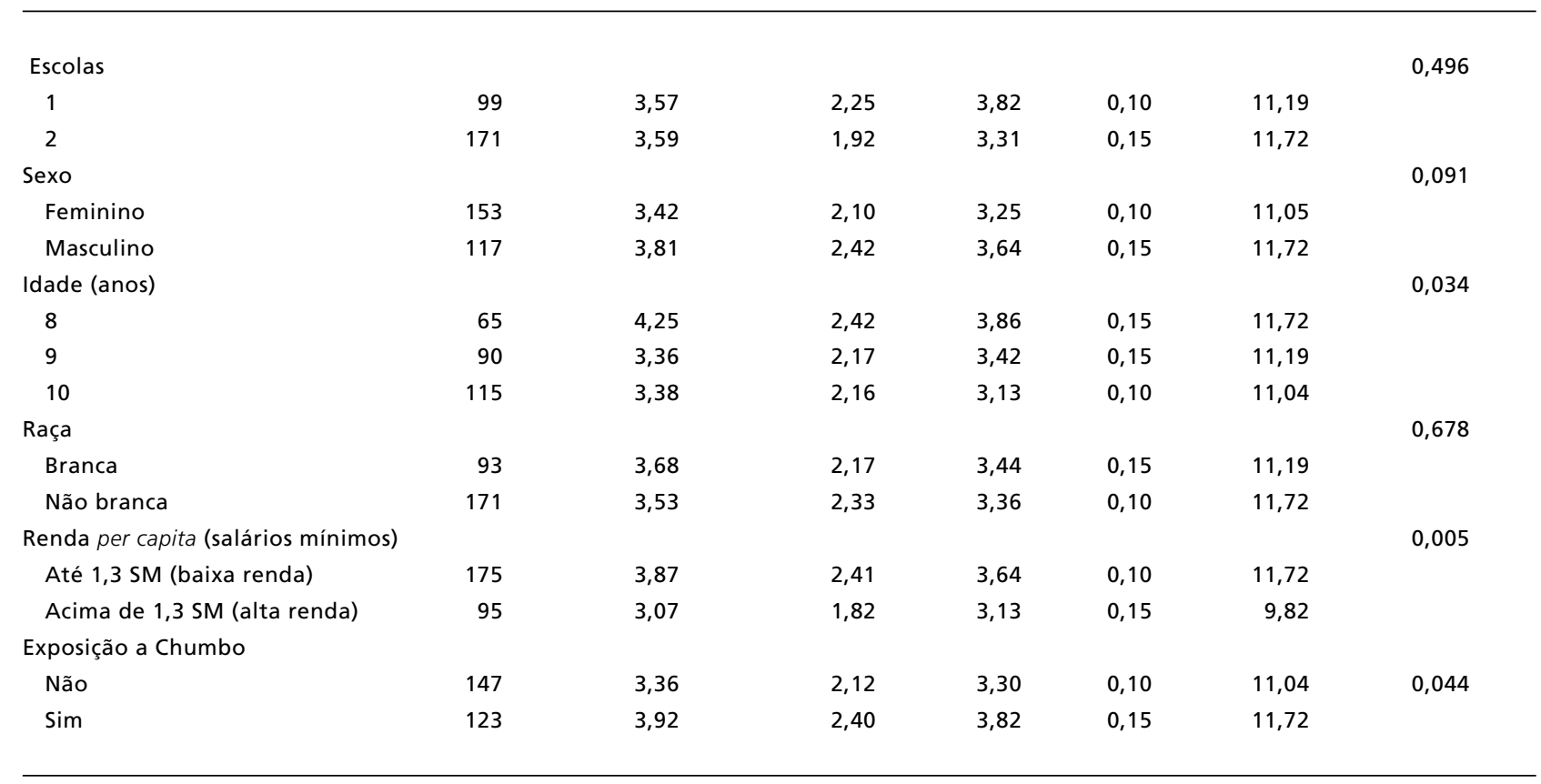

* teste ANOVA; Escola 1= CIEP Yuri Gagarin (Bonsucesso); Escola 2= Escola Desembargador Ney Palmeiro (Jacarepaguá).

Tabela 4

Parâmetros obtidos na regressão linear múltipla.

\begin{tabular}{lcccc}
\hline & $\boldsymbol{\beta}$ & $\mathrm{IC} 95 \%$ & $\begin{array}{l}\mathbf{p} \text { valor do } \\
\text { parâmetro }\end{array}$ & $\begin{array}{l}\text { Coeficiente de } \\
\text { determinação }\end{array}$ \\
\hline Idade & $-1,74$ & $-3,21 ;-0,02$ & 0,021 & \\
Renda média per capita & $-3,52$ & $-5,97 ;-1,07$ & 0,005 & 0,61 \\
Status de exposição & 2,19 & 1,$80 ; 4,56$ & 0,007 & 0,01 \\
\hline
\end{tabular}


Tabela 5

Prevalência de crianças classificadas como expostas segundo escola ( $N=270)$.

\begin{tabular}{|c|c|c|c|c|c|c|c|}
\hline \multirow[t]{3}{*}{ Escola } & \multicolumn{6}{|c|}{ Status de exposição } & \multirow[t]{3}{*}{$\boldsymbol{p}^{*}$} \\
\hline & \multicolumn{2}{|c|}{ Não } & \multicolumn{2}{|c|}{ Sim } & \multicolumn{2}{|c|}{ Total } & \\
\hline & $\mathrm{n}$ & $\%$ & $\mathrm{n}$ & $\%$ & $\mathrm{n}$ & $\%$ & \\
\hline CIEP Yuri Gagarin & 42 & 42,5 & 57 & 57,5 & 99 & 100,0 & \\
\hline $\begin{array}{l}\text { Escola Desembargador } \\
\text { Ney Palmeiro }\end{array}$ & 105 & 61,4 & 66 & 38,6 & 171 & 100,0 & 0,004 \\
\hline Total & 147 & 58,1 & 123 & 41,9 & 270 & 100,0 & \\
\hline
\end{tabular}

* qui-quadrado de Pearson.

\section{Discussão}

Vários fatores são importantes para explicar a exposição ambiental ao chumbo, entre eles o padrão alimentar, os índices de poluição ambiental e os indicadores de qualidade de vida (vulnerabilidade).1012 A Escola 1, onde foram encontradas as maiores concentrações de chumbo em sangue capilar $(75,8 \%$ das crianças no maior quartil de exposição), está localizada no bairro de Bonsucesso, uma área de intensa urbanização, com carência de áreas verdes, grande densidade demográfica (aproximadamente $2,285 \mathrm{hab} / \mathrm{m}^{2}$ ) e próxima às principais vias de tráfego de acesso à cidade. Além disso, o bairro caracterizase pela presença de complexos industriais e comerciais, a segunda maior concentração de veículos, de indústrias e de fontes emissoras de poluentes do município, apresentando uma qualidade do ar classificada como inadequada por conter altas concentrações de material particulado entre outros poluentes. $13-15$

O presente estudo descreve padrões de exposição considerados abaixo do limite de segurança estabelecido pelo CDC. Entretanto, recentes estudos apontam que concentrações de chumbo sanguíneo inferiores a $10 \mu \mathrm{g} / \mathrm{dL}$ podem estar associadas com déficits no desenvolvimento neuropsicológico e, concentrações sanguíneas inferiores a $5 \mu \mathrm{g} / \mathrm{dL}$, podem ser responsáveis por déficits cognitivos, retardo no desenvolvimento emocional e comportamental, decréscimo nos níveis de QI e déficit de memória. 16-18

Cabe ressaltar que, embora os níveis encontrados estejam abaixo do limite de segurança do Centers for Disease Control and Prevention (CDC), ele se encontra mais alto que em grandes estudos anteriores realizados em outros países. Por exemplo, quando comparados os valores médios dos níveis de chumbo do presente estudo com o apresentado pela Fourth
National Report on Human Exposure to Environmental Chemicals, observa-se que ele encontra-se acima dos valores encontrados pelo inquérito de exposição a substâncias químicas americano. Entre os principais resultados apontados por este inquérito, conduzido pelo CDC nos EUA, encontra-se a redução nas concentrações médias de chumbo em sangue de crianças, com idades entre $0 \mathrm{e}$ 5 anos, de 2,23 $\mu \mathrm{g} / \mathrm{dL}$, nos anos de 1999-2000, para $1,51 \mu \mathrm{g} / \mathrm{dL}$, nos anos de 2007-2008, uma redução de $32,28 \%$. Nos dois momentos de investugação do survey os valores encontraram-se inferiores ao encontrado pelo presente estudo $(3,59 \mu \mathrm{g} / \mathrm{dL}) .19$ Especula-se, que o padrão de consumo de crianças seja diferente entre Brasil e Estados Unidos, principalmente pela exposição a produtos de consumos típicos da infância, como brinquedos e alimentos que usem o chumbo como elemento para confecção dos mesmos, bem como a exposição ambiental que não é exclusiva de crianças, como o uso do chumbo como aditivo de combustíveis. Vale lembrar que estudos recentes apontam para a queda nas concentrações de chumbo sanguíneo na população de muitos países, incluindo os Estados Unidos, Austrália, México, Alemanha, Polônia, Suécia, Inglaterra e Índia, que pode ser explicada pelo declínio na contribuição do chumbo ambiental na exposição humana ao metal. Este declínio, no entanto, pode ser o resultado de fatores como a retirada do chumbo da gasolina. ${ }^{19-21}$

Crianças com oito anos de idade apresentaram concentrações médias de chumbo em sangue capilar $(4,25 \mu \mathrm{g} / \mathrm{dL})$ superiores às encontradas em crianças com $9(3,36 \mu \mathrm{g} / \mathrm{dL})$ e dez anos de idade $(3,38$ $\mu \mathrm{g} / \mathrm{dL})$, com diferença estatisticamente significativa $(p=0,034)$. Em geral, as diferenças nos resultados observados nas concentrações de chumbo por idade são devidas a padrões comportamentais e fisiológicos distintos. Destaca-se, ainda o comportamento de crianças mais novas com relação a uten- 
sílios que possuam maior concentração de chumbo em corantes, como lápis de cor, massas de modelar e pinturas de brinquedos de plástico. Esta diferença no padrão de exposição para crianças mais jovens é corroborada por Richardson. ${ }^{10}$ Neste estudo, 195 crianças com idades entre três e oito anos, residentes na cidade de Dublin, tiveram as suas concentrações de chumbo em sangue capilar dosadas. Os resultados obtidos mostraram que as concentrações de chumbo eram significativamente superiores nas crianças com idades entre três e cinco anos quando comparadas com as concentrações encontradas nas crianças com idades entre seis e oito anos. Segundo o autor, este resultado pode ser justificado por hábitos como levar a mão e objetos à boca que podem levar a ingestão de partículas de solo contaminado pelo metal.

Heinzel et al.22 e a Fourth National Report on Human Exposure to Environmental Chemicals, 19 realizada pelo CDC no ano de 2009 , observaram que as crianças que estudavam em escolas situadas em áreas supostamente mais poluídas, apresentavam maiores concentrações de chumbo, embora dentro dos níveis de normalidade. Ozden et al. 23 realizaram um estudo com 760 crianças, com idades entre $11 \mathrm{e}$ 13 anos, provenientes de 13 escolas localizadas em duas áreas distintas da cidade de Istambul (uma área próxima às principais rodovias da cidade e a outra localizada longe destas rodovias). As crianças que estudavam na escola localizada próxima às grandes rodovias da cidade, apresentaram níveis de chumbo sanguíneo acima do valor médio encontrado na população de estudo $(8,4 \mu \mathrm{g} / \mathrm{dL})$.

Indicadores de qualidade de vida que englobam fatores como renda, escolaridade, cultura, acesso a

\section{Referências}

1. Moreira FR, Moreira, JC. A cinética do chumbo no organismo humano e sua importância para a saúde. Ciênc Saúde Coletiva. 2004; 1: 167-81.

2. Mazoto ML, Filhote MIF, Câmara VM, Asmus CIRF. Saúde Ambiental Infantil: Uma revisão de propostas e perspectivas. Cad Saúde Coletiva. 2011; 19 (1): 41-50.

3. Mello-da-Silva CA, Fruchtengarten L. Environmental chemical hazards and child health. J Pediatr. 2005; 81 (Suppl. 5): S205-11.

4. Ahamed M, Verma S, Kumar, A, Siddiquiu MK. Environmental exposure to lead and its correlation with biochemical indices in children. Sci Total Environ. 2005; 346: 48-55.

5. KollerK, Brown T, Spurgeon A, Levy L. Recent developments in low-level lead exposure and intellectual impairment in children. Environ Health Perspect. 2004; 112: 98794.

6. Gump BB, Stewart P, Reihman J, Lonky E,Darvill T, serviços de saúde e saneamento precisam ser considerados quando analisamos os possíveis efeitos à saúde da exposição a poluentes ambientais. $\mathrm{O}$ bairro de Bonsucesso encontra-se situado abaixo do bairro de Jacarepaguá no ranking dos indicadores de qualidade de vida, em particular com relação à renda e à escolaridade, o que representa uma condição de vulnerabilidade em relação a exposição ambiental ao chumbo. $24-27$

Neste estudo, que apresenta a especificidade de coleta de sangue capilar como amostra biológica, observou-se uma diferença estatisticamente significativa entre as concentrações de chumbo em sangue capilar divididas por quartis de distribuição das escolas estudadas que poderia ser explicada pelas condições de exposição ambiental onde as duas escolas estão localizadas.

É importante ressaltar que foi observada a existência de uma lacuna na construção do conhecimento sobre a toxicidade do chumbo em populações que apresentam características semelhantes às do estudo, o que tornam relevantes os resultados encontrados, bem como fundamental o estímulo à continuidade das avaliações e a proposição de novos estudos que consolidem os achados dos programas de monitoramento humano da exposição a substâncias químicas.

\section{Agradecimentos}

Ao Ministério da Saúde pelo financiamento da pesquisa e à Universidade de São Paulo/Ribeirão Preto pelas análises toxicológicas.
Matthews KA, Parsons PJ. Prenatal and early childhood blood lead levels and cardiovascular functioning in $91 / 2$ year old children. Neurotoxicol Teratol. 2005; 27: 655-65.

7. Ballew C, Khan LK, Kaufmann R, Mokdad A, Miller DT, Gunter EW. Blood Lead Concentrations and Children's Anthropometric Dimensions in the Third National Health and Nutrition Examination Survey (NHANES III), 198894. J Pediatr. 1999; 134 (5): 623-30.

8. Jain NB, Laden F, Guller U, Shankar A, Kazani S, Garshick E. Relation between blood lead levels and childhood anemia in India. Am J Epidemiol. 2005; 161 (10): 968-73.

9. Batista BL, Rodrigues JL, Nunes JA, Souza VC, Barbosa F Jr. Exploiting dynamic reaction cell inductively coupled plasma mass spectrometry (DRC-ICP-MS) for sequential determination of trace elements in blood using a dilute-andshoot procedure. Analytica Chimica Acta. 2009; 639: 13-8.

10. Richardson RM. Blood lead concentrations in three to eight year old school-children from Dublin city and rural country wicklow. Irish J Med Sci. 2008; 151 (1): 203-10. 
11. Schimitt N, Philion JJ, Larsen AA, Harnadek M, Lynch AJ. Surface soil as a potencial source of lead exposure for young children. CMA J. 1979; 121 (8): 1474-8.

12. Mielke HW, Reagan PL. Soil is an important pathway of human lead exposure. Environ Health Perspect. 1998; 106 (1): $217-22$.

13. Burgoon DA, Rust SW, Hogan KA. Relationship among lead levels in blood, dust and soil. In: Lead poisoning: exposure abatement regulation. Breen JJ, Stroup CR, Eds. Boca Raton, FL: Lewis Publishers. 1995; p. 255-64.

14. Prefeitura da Cidade do Rio de Janeiro. Secretaria Municipal de Urbanismo. Coleção Estudos da Cidade. Rio Estudos; 2003.

15. Instituto Estadual do Ambiente. Relatório Anual da Qualidade do Ar do Estado do Rio de Janeiro. INEA; 2009.

16. CDC (Centers of Disease Control). Preventing lead poisoning in young children: a statement by the Centers for Disease Control. U.S. Department of Health and Human Services, Public Health Service, Atlanta, GA; 1991.

17. Olivero-Verbel J, Duarte D, Echenique M, Guette J, Johnson-Restrepo B, Parsons AP. Blood lead levels in children aged 5-9 years living in Cartagena, Colombia. Sci Total Environ. 2007; 372: 707-16.

18. Moreno ME, Acosta-Saavedra LC, Meza-Figueroa D, Vera E, Cebrian ME, Ostrosky-Aranda ES. Biomonitoring of metal in children living in a mine tailings zone in southern Mexico: a pilot study. Int JHyg Environ Health. 2010; 213: $252-8$

19. CDC (Centers of Disease Control). Fourth National Report on Human Exposure to Environmental Chemicals. Department of Health and Human Services Centers of Disease Control and Prevention. Atlanta, GA; 2009.
20. Ahamed M, Siddiqui MKJ. Environmental lead toxicity and nutritional factors. Clin Nutr. 2007; 26: 400-8.

21. Ahamed M, Verma S, Kumar A, Siddiqui MK. Blood lead levels in children of Luxknow, India. Environ Toxicol. 2010; 25: 48-54.

22. Heinzel I, Gross R, Stehle P, Dillon D. Assessment of lead exposure in schoolchildren from Jakarta. Environ Health Perspect. 1998; 106: 499-501.

23. OzdenTA, Kiliç A, Toparlak D, Gokçay G, Saner G. Blood lead levels in school children. Indoor Built Environ. 2004; 13: $149-54$

24. Silva OMP, Pachoca LA. Contribuição da vulnerabilidade na determinação do Índice de Desenvolvimento Humano: estudando o Estado de Santa Catarina. Ciênc Saúde Coletiva. 2007; 12 (5): 1209-19.

25. RobertsJR, Hulsey TC, Curtis GB, Reigart JR. Using geografic information system to assess risk for elevated blood lead levels in children. Public Health Reports. 2003; 118: 221-9

26. Minayo MCS, Hartz ZMA, Buss PM. Qualidade de vida e saúde: um debate necessário. Ciênc Saúde Coletiva. 2000; 5 (1): 7-18

27. Herculano SC. A qualidade de vida e seus indicadores. Ambiente Soc. 1998; 2: 77-99.

Recebido em 22 de maio de 2014

Versão final apresentada em 22 de agosto de 2014

Aprovado em 30 de setembro de 2014 